\section{Commentary on How Could I Have Done This?" Thoughts of Mothers of Children with Fetal Alcohol Syndrome}

Received: September 09, 2017; Accepted: September 23, 2017; Published: September 30, 2017

Biological mothers who give birth to children with Fetal Alcohol Syndrome are an underserved and under-researched population [1]. Increasing our understanding of the thoughts and feelings of biological mothers of children with FAS may enable us to assist those women who are coming to terms with drinking alcohol during their pregnancies and may help us to screen and identify women at the greatest risk of having a child with FAS and provide prevention services. While working and researching this population, this brief article provides thoughts and recommendations for those that encounter women who consumed alcohol during pregnancy.

All of the mothers interviewed found a way to understand and frame their drinking (whether before, during or after their pregnancies) for themselves in a way that included: utilization of the disease model of addiction, recalling being told inaccurate or incomplete information of the danger of taking alcohol during pregnancy, following the advice of others (family members, friends, physicians) and stating that they were initially unaware of their pregnancy when they were drinking [1]. In addition, this study has potentially important implications for practice.

\section{Mental Health Professionals}

\section{Biological mothers}

Due to the complexity of the parenting relationship, understanding and supporting this group of mothers is necessary. A mother may face guilt, especially if she has recently learned of the diagnosis as participants noted a strong and pervasive sense of guilt [1]. Practitioners should be advised to assess for guilt and its effects and if indicated to work with the mother around improving support and coping during times of increased feelings of guilt. The mental health practitioner can also be helpful in guiding the mother towards an explanation or understanding of her actions. However, it must be noted that this was a small study, therefore a larger number of explanations (such as: mental health issues include psychotic disorders, coercion by partner or family members) may exist and larger samples need to be studied to determine if there are other such explanations.

Referral to the National Organization on Fetal Alcohol Syndrome (NOFAS) website (http://www.nofas.org) may be a useful

\author{
Joelle Zabotka* \\ School of Social Work, Monmouth \\ University, USA
}

*Corresponding author: Joelle Zabotka

झ jzabotka@monmouth.edu

PhD, LCSW, LCADC, Assistant Professor, Coordinator-Clinical Practice with Families and Children Concentration, School of Social Work, Monmouth University, USA.

Citation: Zabotka J (2017) Commentary on How Could I Have Done This?" Thoughts of Mothers of Children with Fetal Alcohol Syndrome. J Drug Abuse Vol.3 No.3:21 recommendation by clinicians to their clients as a source of greater information and support. The NOFAS Birth Mothers Network is a source of support to any women who consumed alcohol during pregnancy and may have a child affected by prenatal alcohol exposure and this support is provided by women who have been through similar circumstances. The Birth Mothers Network offers peer support and mentoring, referrals for FAS evaluation/ diagnosis and addiction treatment services, a monthly conference call and attendance at an annual retreat (expenses paid) (www. nofas.org). Mothers offer support to each other through email, phone and some in-person contact. This group of mothers supports each other through addiction recovery (if applicable) and/or through the challenges of parenting a child suffering the effects of prenatal alcohol exposure. The mentoring system can be a great source of support, and an opportunity for experienced mothers to share their wisdom, successes and failures with families of newly diagnosed children [2]. Mothers participating in this study noted feelings of judgment and anger when attending support groups also attended by adoptive/foster parents of children with FAS. Support/information groups specifically for birth mothers may create a more comfortable, nonjudgmental and accepting environment. Through support services specific to birth mothers, information about characteristics of FAS, parenting techniques and suggestions can be disseminated. In addition, topics such as prevention of alcohol exposure during subsequent pregnancies, recovery, stigma and guilt could be included as well. Although the benefits of NOFAS Birth Mothers Network are clear, it may not be a good fit for every birth mother. The establishments and/or utilization of local support groups 
may be an effective way to further assist such mothers and their children.

Clinicians encountering women with a child with FAS should pay particular attention to their own thoughts and feelings around bias and judgment. Clinicians may find themselves feeling angry or have an impulse to shame the mother. This is understandable given that the child has been harmed by the mother's actions. However, this research begins to demonstrate that this group of women were trapped by addiction, overwhelmed by their own histories, and/or misinformed by friends and family members; they did not feel callous or uncaring toward their unborn children [1]. There was never intent to harm and clinicians should keep this in mind as they attempt to empathize and assist their clients. Clinicians should search for more information and seek quality clinical supervision around these difficult, emotionally-charged issues.

\section{Addictions specialists}

Addictions specialists play an important role in both treatment of women and prevention of future occurrences of FAS. Every woman of fertility age should be screened and educated about the dangers of drinking while pregnant or before becoming pregnant and addictions specialists need to clearly and emphatically communicate to their patients that there is no safe amount of alcohol during a pregnancy [3].

This recommendation is in light of the finding that several of the participating mothers said that they perceived that family members, friends and even physicians supported their drinking during pregnancy. Also, addiction programs can offer education about the dangers of drinking alcohol during pregnancy to both men and women receiving addictions treatment. It is never too early to provide accurate information to both adolescents and adults receiving addictions treatment. Women should not

\section{References}

1 Zabotka J, Bradley C, Escueta M (2017) "How could I have done this?" Experiences of birth mothers of children diagnosed with fetal alcohol syndrome. J Soc Work Pract Addict 17: 258-274. bear the burden of helping to ensure a healthy pregnancy and baby alone. Their partners and expecting fathers should have the same education and information in order to offer support and assistance. Several participating mothers perceived that their family members, friends, and even their physicians gave permission for alcohol intake during pregnancy. Addictions professionals are a good source for accurate information that can clarify misinformation and discourage thoughts and intentions of drinking during pregnancy.

It is critically important that accurate and clear information is included in the education of addictions professionals, mental health practitioners and social workers. These professionals must be clear that there is no safe amount of alcohol to take during pregnancy to assist in prevention of future occurrences of FAS. In addition, information on indicators of Fetal Alcohol Syndrome, including facial features and neurobehavioral symptoms should be included in the curricula of these groups of professionals so that they may serve as screening and referral sources. Finally, the addiction and mental health professionals must have information on the behavioral and psychological outcomes of the FAS diagnosis, such as the results of this study, so that they may have the knowledge to assist this population of mothers and children.

\section{Implications for policy}

Continued public education must be funded and targeted to society at large including not only to pregnant women, but all women and men as findings demonstrate that many pregnant women rely on the advice of family and friends. There has been a greater push for public education, such as public education warnings in bars, liquor stores and restaurant bathrooms, but it must be broadened, for example, to include billboards, sides of buses, public service announcements of television and radios and bigger and bolder warnings on alcohol containing products.
2 Devries J, Waller A (2004) Fetal alcohol syndrome through the eyes of parents. Addict Biol 9: 119-126.

3 Van der Zee B, de Beaufort I, Temel S, de Wert G, Denktas S, et al. (2011) Prconception care: An essential prevention strategy to improve children's and women's health. J Public Health Policy 32: 367-379. 